PART I

\title{
SPIRAL STRUCTURE IN GALAXIES
}




\title{
2. SPIRAL STRUCTURE IN EXTERNAL GALAXIES
}

\author{
W. W. MORGAN \\ Yerkes Observatory, Williams Bay, Wisc., U.S.A.
}

\begin{abstract}
The need for observing at least four different categories of optical objects for a satisfactory definition of optical spiral structure is emphasized. A sequence of optical form-types for the description of spiral structure is outlined. A pronounced difference in the nature of the spiral structure of the outer and inner parts of Messier 33 is illustrated. Striking local differences between spiral arms defined by differing criteria are noted.
\end{abstract}

\section{Introduction}

There are at least four different categories of optical objects which can be considered to define spiral arms: (a) Hil regions, (b) dust lanes, (c) blue supergiants (with a certain admixture of $h-\chi$ Persei-type red supergiants), and (d) a non-blue stellar population which can be observed on plates exposed to the yellow spectral region. The appearance - and even location - of the arms depicted varies somewhat on passing from one category to another.

The Hil regions define arm segments which are narrow, somewhat irregular in shape, and which may be relatively short in length. The arms as defined by dust segments tend to be narrow also, may be quite regular in the inner parts of the main body, and sometimes coincide in position with the arm segments as defined by the $\mathrm{H}$ II regions. The brightest resolved blue stars (and red supergiants of the h- $\chi$ Perseitype) lie in arm-like segments somewhat less sharply defined than those of the first two categories. The 'non-blue star-arms' tend to have the smoothest and most regular appearance in the inner parts of spirals. The local width of the arms increases progressively from the $\mathrm{HII}$ region type (the narrowest), to the non-blue star-arms (the widest).

From these circumstances it can be seen that if we wish to discuss the general phenomenon of spiral structure we must describe in detail, and describe separately, the spiral arms as shown by the various categories of objects. Only in this way will we be able to bring full observational weight to bear on the definition and evolution of extragalactic spiral structures. In addition, it is only by such a complex procedure that we will be able to compare with precision the spiral structure of our own Galaxy with that of external galaxies. There is no single indicator capable of defining completely the general phenomenon called 'spiral structure'; it is only by a collation of the results from the various categories of objects (including those in radio astronomy) that a satisfactory description of spiral structure can be arrived at.

\section{The Frame of Reference}

The optical form-classification of Hubble in its 1935 state is described in Hubble's 
(1936) The Realm of the Nebulae. The normal spirals ( $\mathrm{Sa}, \mathrm{Sb}, \mathrm{Sc}$ ) are illustrated in the famous tuning fork diagram; the two principal criteria for their classification were: (1) the 'relative luminosity of nuclear region and spiral arms', and (2) the 'openness of the arms'. An inspection of Hubble's (1936) diagram suggests that the principal discriminant between classes $\mathrm{Sa}$ and $\mathrm{Sb}$ was the openness of the arms, since the relative luminosity of the nuclear region to the spiral arms in the sketches of classes Sa and $\mathrm{Sb}$ is not very different.

The practical result of this weighting of the two criteria on later classification work on the Hubble system (Humason et al., 1956; Sandage, 1961) has been to create an Sa class which includes objects exhibiting a wide range in the nuclear concentration of luminosity - and a pronounced overlap between classes $\mathrm{Sa}$ and $\mathrm{Sb}$ in this same characteristic. From this it is suggested that the sequence $\mathrm{Sa}-\mathrm{Sb}-\mathrm{Sc}$ is not a completely one-dimensional sequence, since the two principal classification criteria can give conflicting Hubble types. The Hubble Sc type includes some Yerkes types in the range aI-gS; the Hubble type Sb includes some Yerkes types in the range fgS-kS. As shown in Figure 1, the Hubble Sa class may parallel class Sb with respect to the Yerkes onedimensional stellar-population form-sequence. Six standard galaxies are reproduced in Figures 2 and 3; they are from Mount Wilson 60-inch and 100-inch plates.

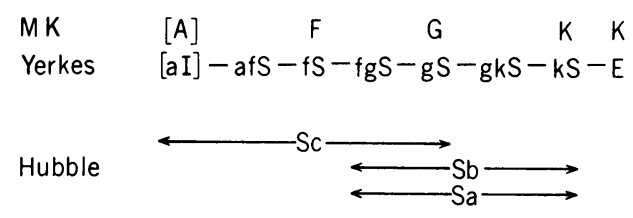

Fig. 1. A comparison of Yerkes form classification with blueviolet spectral types, and Hubble types for spirals. The $\mathrm{Sb}$ and $\mathrm{Sa}$ classes overlap with respect to spectral type of the nuclear region.

A relationship somewhat similar to Figure 1 can be set up for the barred spirals.

The Yerkes form types (based on the relative luminosity of the nuclear region to the main body) are fairly well correlated with the integrated spectral type of the nuclear region. The greatest stellar contribution in the blue-violet to the luminosity of the nuclear region progresses from A stars (4490, Sc, aI) to K giants (2841, Sb, kS). No spectrogram was available for 4535 . The populations in the later parts of this sequence appear to be of the ordinary strong metallic-line type. We may label the above Yerkes sequence as an evolutionary sequence for the stars contributing most to the luminosity of the inner region of the galaxies concerned.

There is a wide variation in the detailed optical aspects of galaxies classified in each category of the Yerkes system; the criterion used has been the relative luminosity of the nuclear region to that of the remainder of the main body; the only added criterion was that of visible spiral structure. NGC 4490 , classified on the Hubble system as Sc, has been considered to belong to the Irregular class on the Yerkes system; however, this is a delicate distinction; and the galaxy may become a prototype for a category described by G. R. Burbidge and by Morgan, and labelled by them 


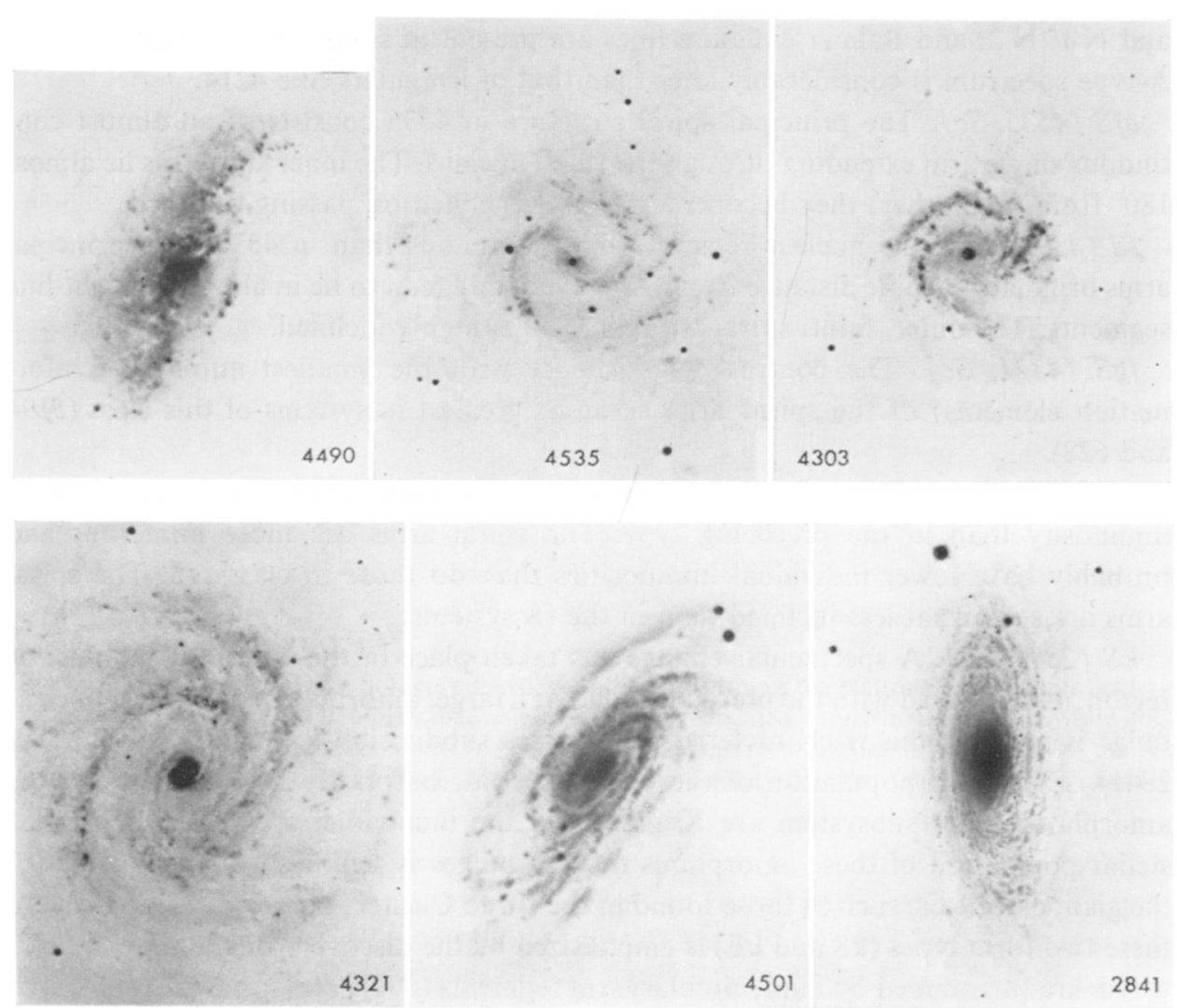

Fig. 2 and 3. A series of six standard galaxies illustrating Yerkes form types for spirals. NGC 4490 (aI; Sc) has an A-type absorption spectrum. The sequence 4535, 4303, 4321, and 4501 is in order of increasing luminosity of the inner region, relative to that of the main body as a whole. NGC 2841 possesses a large, luminous, nuclear subsystem; the latter is entirely different in appearance from the other galaxies illustrated; the light of the inner region in the blue-violet is due principally to yellow giants. The inner spiral arms (dust or stars) tend to be rather highly inclined for all except 2841 . Mount Wilson 60-100-inch photographs.

'integral signs'; such objects may turn out to be closely related in nature to such systems as 5247, and objects Nos. 31 and 33 in the Arp Atlas of Peculiar Galaxies.

The earliest-type object with well-developed spiral structure has been classified as afS (4535). In the series illustrated, the spirals 4303, 4321, 4501 and 2841 form a sequence of progressively more luminous inner regions - and also of progressively later spectral type in blue-violet wavelengths.

\section{The Phenomena of Spiral Structure as a Function of Yerkes Form-Type}

aI $(4490, S c)$. This might be considered as a galaxy consisting of a long, distorted, very broad spiral arm. [O II] 3727 is outstanding in strength in the central region; 
and $\mathrm{N} 1, \mathrm{~N} 2$, and Balmer emission lines are present in some regions. However, the A-type spectrum is considerably later than that of irregulars like 4214 .

afS $(4535, S c)$. The principal spiral structure in 4535 consists of an almost continuous single arm extending through the small nucleus. The inner star arms lie almost $180^{\circ}$ from each other; they become less highly inclined on passing outward.

$f S(4303, S c)$. The nuclear region is more luminous than in 4535 . The principal arms originate at some distance from the nucleus and tend to lie in almost straight-line segments. The outer, faint, spiral structure is less highly inclined.

$f g S(4321, S c)$. The contrast (or visibility with the smallest number of information elements) of the spiral arms is at its greatest in systems of this class (5194 and 628).

$g S(4501, S c)$. The entire inner region of the main body has higher relative luminosity than in the preceding type. The spiral arms are more numerous and probably have lower individual luminosities than do those in class fgS. The spiral arms are somewhat less inclined than in the fS systems.

$k S(2841, S b)$. A spectacular change has taken place in the nature of the nuclear region, as compared with the preceding spirais: a large, amorphous, luminous, nuclear bulge is present; this is characteristic of Yerkes subdivision kS (M 31, 4594, M 81, 2841). The stellar population of the principal contributors to the luminosity of the amorphous inner subsystem are $\mathrm{K}$ giants (in the blue-violet spectral region). The stellar population of these amorphous nuclear bulges is strikingly similar to that of the giant ellipticals, such as those found in the Virgo Cluster; the relationship between these two form types ( $\mathrm{kS}$ and $\mathrm{kE}$ ) is emphasized by the discovery of certain ellipticals which are surrounded by faint, circular, arm segments (474). The spiral arms observed in 2841 are of low inclination and of considerably lower contrast and luminosity than in the fg spirals.

\section{The General Large-Scale Characteristics of Spiral Arms}

The inclination of the inner arms of $\mathrm{fS}$ and fgS galaxies tends to be high; that of the spiral arms of the $\mathrm{kS}$ galaxies tends to be considerably lower, and not far from circular. The integrated MK spectral types of the nuclei of $\mathrm{fS}$ and fgS galaxies are of class $\mathrm{F}$ in the blue-violet region; the nuclear bulges of $\mathrm{kS}$ galaxies are of MK spectral class $\mathrm{gK}$. A relationship between the average inclination of the inner arms and the average evolutionary state of the most luminous fraction of the nuclear stellar population is therefore suggested.

Figure 4 shows the outer part of M 33 from the blue print of the National Geographic Society-Palomar Sky Survey. The positions of the principal two inner star arms are marked from Yerkes photographs by Dr. Robert Garrison. The complexity in number of the outer arms is in contrast to the pair of principal inner arms.

Figure 5 illustrates two photographs of M 101 obtained by Garrison at Yerkes. A section of an $\mathrm{HII}$ arm that appears to be separated from the nearest strong star arm is marked. 


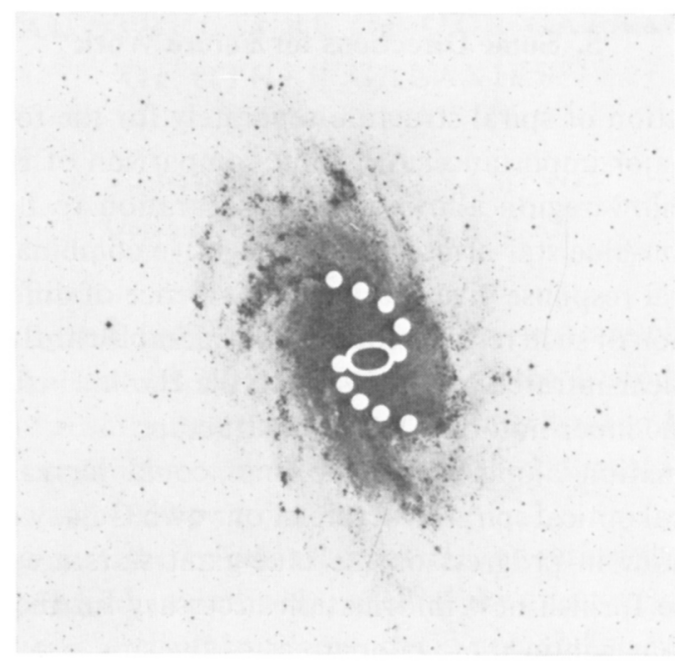

Fig. 4. The spiral M 33 (fS; Sc) reproduced from the blue print of the National Geographic SocietyPalomar Sky Survey. The two principal inner star arms are located approximately by the white dots. The outer spiral structure can be seen to be complex and to a certain extent independent of the two principal arms. Copyrighted by the National Geographic Society-Palomar Observatory Sky Survey.
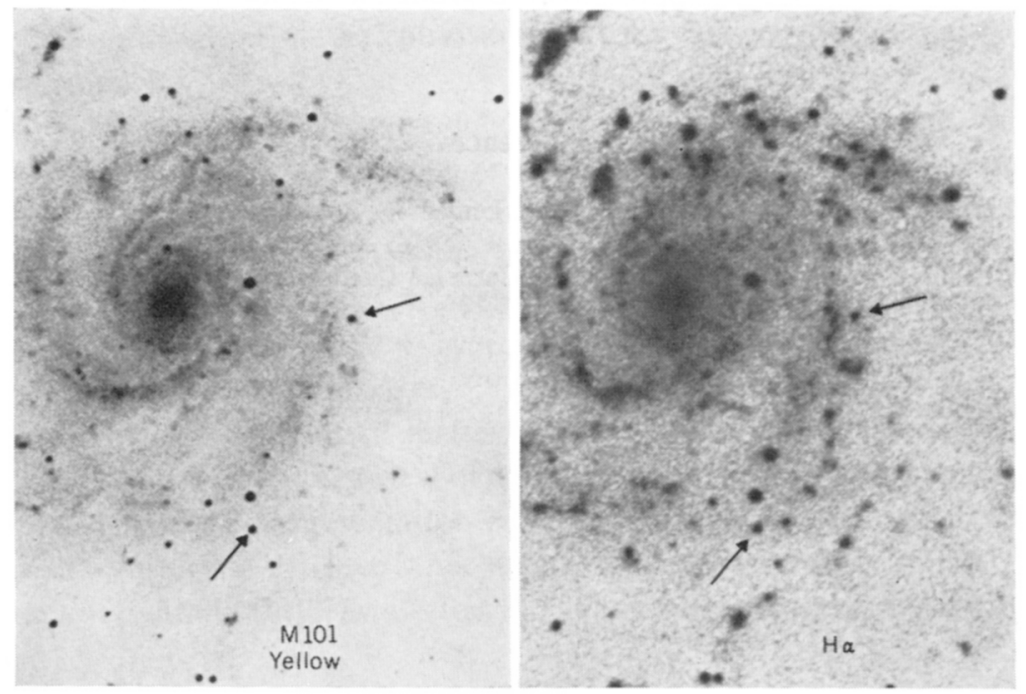

Fig. 5. Yellow and $\mathrm{H} \alpha$ photographs of $\mathrm{M}$ 101. On the yellow plate, one of the principal star arms can be seen extending slightly to the left of the two stars marked. On the $\mathrm{H} \alpha$ plate a string of $\mathrm{HII}_{\mathrm{II}}$ regions can be seen slightly to the right of a line joining the two marked stars. This can be described as a 'splitting' of an arm segment, with the two segments differing in physical constitution and width. Yerkes photographs by Robert Garrison. 


\section{Some Directions for Future Work}

The detailed delineation of spiral structure separately for the four optical indicators listed above is of major importance. A careful comparison of $\mathrm{H} \alpha$ photographs with exposures in the yellow region allows a clean separation to be made between the $\mathrm{H}$ II arms and the non-blue star-arms. Positive-negative combinations of plates taken with differing spectral response give spectacular evidence of differences in arm structure; the interpretation of such results requires considerable care, if unique conclusions are to be drawn. A near-infrared survey in which the $\mathrm{H} \alpha$ line is filtered out could give useful evidence of the inner non-blue star-arm structure.

Additional information along the above lines could increase considerably our knowledge of the local optical spiral structure of our own Galaxy - when spectroscopic parallax programs now in progress on the blue-giant stars are completed. Such an approach would also furnish new information necessary for the collation of optical and radio observations within three kiloparsecs of the sun.

\section{Acknowledgments}

We are indebted to Dr. H. W. Babcock, Director, for permission to reproduce the six Mount Wilson photographs of Figures 2 and 3, from 60-inch and 100-inch originals and for permission to reproduce Figure 4 from the National Geographic SocietyPalomar Sky Survey. The photographic work was carried out by Mr. J. W. Tapscott.

We wish to acknowledge support from a grant on Stellar Classification from the National Science Foundation.

\section{References}

Hubble, E.: 1936, The Realm of the Nebulae, Yale University Press, New Haven.

Humason, M. L., Mayall, N. U., and Sandage, A. R.: 1956, Astron. J. 61, 97.

Sandage, A.: 1961, The Hubble Atlas of Galaxies, Carnegie Institution of Washington, Washington. 